\title{
A Flow Control Technique Utilizing Air Blowing to Modify the Aerodynamic Characteristics of Pantograph for High-Speed Train"
}

\author{
Mitsuru IKEDA $^{* *}$, Kazushige YOSHIDA ${ }^{* *}$ and Masahiro SUZUKI ${ }^{* *}$ \\ ${ }^{* *}$ Railway Technical Research Institute, \\ 2-8-38 Hikari-cho, Kokubunji-shi, Tokyo 185-8540, JAPAN \\ E-mail: suzuki@rtri.or.jp
}

\begin{abstract}
To modify aerodynamic characteristics of pantographs for high-speed trains, we propose to blow air from the surface of a panhead. The outlets for air blowing are bored in rows near the trailing edge of the panhead. To estimate the effect of the blowing, we first conduct a wind tunnel test using a two-dimensional panhead model. The results reveal that the pressure distribution on the model can be changed by the blowing. Then we examine a full-scale pantograph model by a wind tunnel test to prove that the proposed method has a great potential for practical applications.
\end{abstract}

Key words: Pantograph, Optimization, Computational Fluid Dynamics, Wind Tunnel Experiment

\section{Introduction}

Aerodynamic and aeroacoustic characteristics of the pantographs, which are mounted on the roof of the car body as displayed in Fig. 1, have become very important properties for high-speed trains to achieve steady current collecting performance and environmental compatibility ${ }^{(1)}$. These characteristics depend on a number of parameters, such as the train speed, the attack angle variation due to dynamic motion of the pantograph, track conditions (open air or tunnel), and changes of the contact strip configuration caused by its wear.

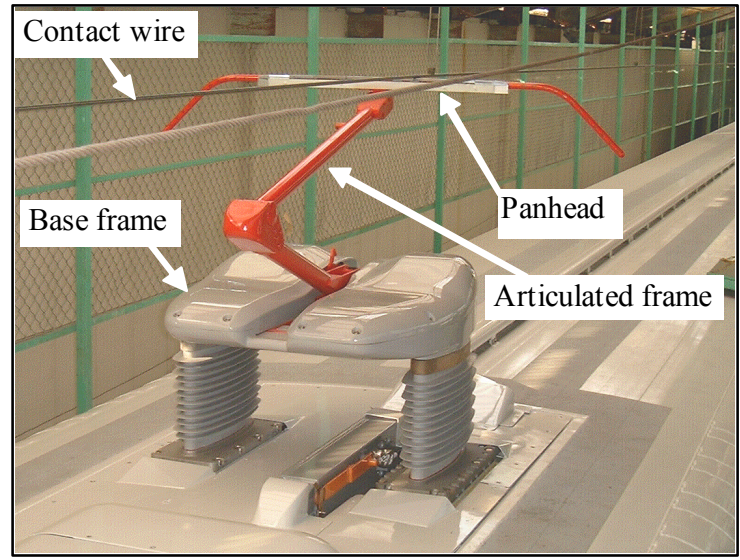

Fig. 1 An example of pantograph for Shinkansen trains and a contact wire 
The lift force acting on the pantograph ought to be within the suitable range, because it affects the contact force between the pantograph and the contact wire. In addition, the aeroacoustic noise generated by the pantograph is one of the main wayside noise sources. It is required to reduce the aeroacoustic noise of the pantograph for further speed up of trains.

The configuration of a panhead, which is set on top of an articulated frame to touch the contact wire, exerts strong influence on the lift force and the aeroacoustic noise of the whole pantograph. Thus, the panhead shape is critical in designing the pantographs for the high-speed trains.

In general, objects of aerodynamically smoothed shape generate lower aeroacoustic noise than bluff objects, but the lift force acting on them is very sensitive to flow conditions such as flow speed, attack angle and changes in the shapes. Hence it is not easy to find out a suitable configuration which can reduce aeroacoustic noise and stabilize the lift force simultaneously.

Recently, in the field of fluid dynamics, various techniques for active flow controls have been studied vigorously to improve the aerodynamic properties. If such techniques can be applied to the panhead, its shape can be designed so as to reduce aeroacoustic noise without any severe constraints on adjusting its lift force. This means that high priority will be placed on the reduction of aeroacoustic noise in panhead designing.

In this study, we applied a flow control technique to a panhead. A wind tunnel test with a two-dimensional panhead model examined the effect of the proposed method. Then a full-scale pantograph equipped with the flow control device has proved efficacy of the technique.

\section{Nomenclature}

$\begin{array}{ll}C_{D}: & \text { Drag coefficient }\left(=2 F_{D} / \rho U^{2} S\right) \\ C_{L}: & \text { Lift coefficient }\left(=2 F_{L} / \rho U^{2} S\right) \\ C_{P}: & \text { Pressure coefficient }\left(=2 P / \rho U^{2}\right) \\ F_{D}: & \text { Drag force }[\mathrm{N}] \\ F_{L}: & \text { Lift force }[\mathrm{N}] \\ L: & \text { Chord length of the panhead }(=0.12[\mathrm{~m}]) \\ S: & \text { Representative area of the two-dimensional panhead } \\ & \quad \text { Pressure }[\mathrm{Pa}] \\ P: & \text { Reynolds number }(=U L / v) \\ \mathrm{Re}: & \text { Free stream velocity }[\mathrm{m} / \mathrm{s}] \\ U: & \text { Span length of the two-dimensional panhead } \quad(=0.6[\mathrm{~m}]) \\ W: & \text { Cartesian coordinates } \\ x, y, z: & \text { Increment of drag coefficient by the air blowing } \\ \Delta C_{D}: & \text { Increment of lift coefficient by the air blowing } \\ \Delta C_{L}: & \text { Increment of pressure coefficient by the air blowing } \\ \Delta C_{P}: & \text { Density of air }\left[\mathrm{kg} / \mathrm{m}^{3}\right] \\ \rho: & \text { Kinematic viscosity }\left[\mathrm{m}^{2} / \mathrm{s}\right]\end{array}$

\section{Flow control by air blowing}

As the active flow control technique, various methods have been proposed ${ }^{(2)}$. There are methods utilizing air blowing among them ${ }^{(3,4)}$. The train is equipped with air compressors and reservoirs for a raising and lowering mechanism of the pantograph, air springs between the car body and the bogies, and so on. Thus the technique with the air 
blowing seems to be applicable to control of the lift force of the pantograph.

In order to change the flow field around bodies by the air blowing technique, time-periodic blowing whose frequency is similar to or larger than the frequency corresponding to the shear layer instability is often exerted ${ }^{(4)}$. It is because the blowing has to interact with the quasi-coherent flow structure near the object surface, in order to control the flow pattern with small power. This method requires an ejection device that can be actuated quickly. However, it is difficult for the pantograph to be equipped with such an advanced device, since a voltage of $25 \mathrm{kV}$ is applied between the pantograph and car body, and a large current (400 A) flows through the pantograph.

If only the steady lift force can be controlled by the steady blowing, however, it is practically useful even if its control response is not so quick. Therefore, we utilize steady air blowing to control the lift force of the pantograph.

\section{Wind tunnel test of two-dimensional panhead model}

\subsection{Two-dimensional panhead model with air blowing}

Figure 2 presents a two-dimensional panhead model used in our wind tunnel test. The model has a $55 \mathrm{~mm}$-thick uniform cross section with a chord length of $120 \mathrm{~mm}$. The span length of the model is $600 \mathrm{~mm}$ to correspond to the dimensions of the wind tunnel nozzle.

An attack angle, which is the angle between the approaching flow and the horizontal center line of the panhead, is set at zero degree. Although panheads are positioned above train roofs horizontally in practical situations, the model is set vertically for ease of set-up. Hereafter the top side refers to the side of the panhead facing in the positive y-direction and the bottom to that facing opposite direction.

The configuration of this model was designed using an optimization method combined with a numerical flow simulation, so as to minimize lift force fluctuations at various attack angles under the conditions of new and worn contact strips ${ }^{(5)}$. In this optimization procedure, a vertically symmetric condition was imposed. The chord length and the minimum thickness of the panhead were also defined as constraints. A wind tunnel experiment proved that the aeroacoustic noise of this panhead is smaller than that of a currently used panhead, whereas its lift force characteristics is similar to that of the currently used one.

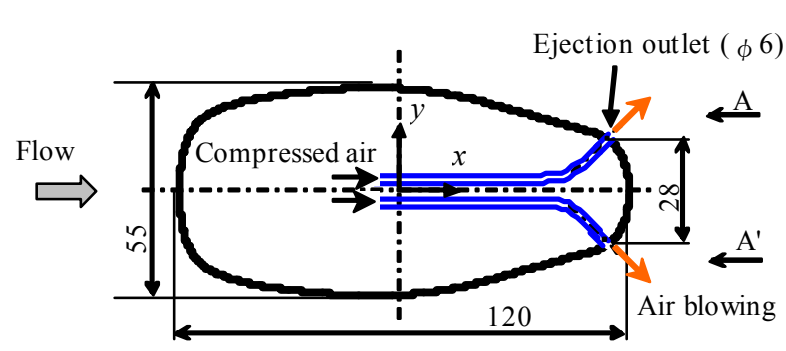

Cross-sectional view

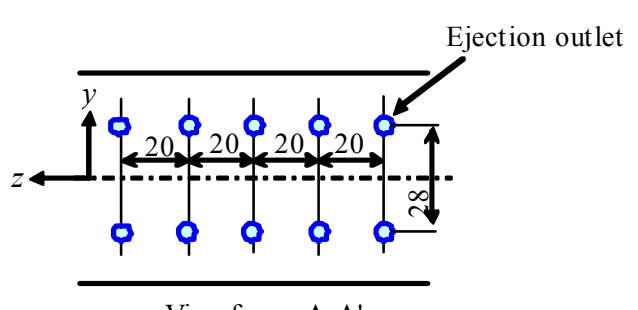

View from A-A'

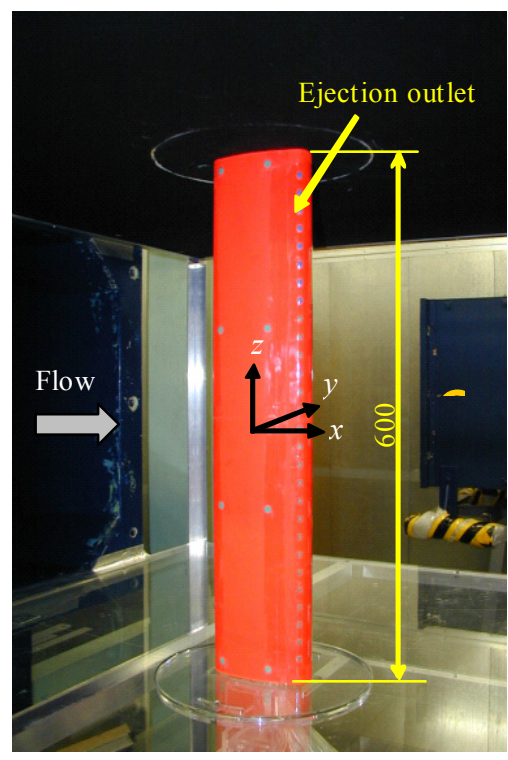

Fig. 2 Two-dimensional model of the panhead with the air blowing (unit: $\mathrm{mm}$ ) 
The model has 56 ejection outlets, each with a diameter of $6 \mathrm{~mm}$; its interval in the span-wise direction is $20 \mathrm{~mm}$. These outlets are located on the top and bottom sides of the model near its trailing edge. The lift force is intended to be controlled by blowing air from single side of the model.

To measure the pressure distribution on the model surface, this model has 45 pressure ports in the intermediate section of the span-wise direction. The brass pipe with an internal diameter of $0.5 \mathrm{~mm}$ is used for each pressure port.

\subsection{Experimental description}

A small-scale anechoic wind tunnel belonging to the Railway Technical Research Institute (RTRI) was used for this test. The dimension of the nozzle is $720 \mathrm{~mm}$ in width and $600 \mathrm{~mm}$ in height. Figure 3 depicts the experimental apparatus used in this wind tunnel test. The panhead model was vertically fitted by balances on both sides to measure lift and drag forces, and two end-plates were installed on top and bottom sides of the test section to maintain a two-dimensional flow. The end-plate measures $1200 \mathrm{~mm}$ in wide and $2000 \mathrm{~mm}$ in length.

The flow velocity was set at $42 \mathrm{~m} / \mathrm{s}(150 \mathrm{~km} / \mathrm{h})$ and the Reynolds number based on the chord length of the model was $3.2 \times 10^{5}$.

Air was supplied to the outlets from the air tank with a volume of $34 l$ located out of the model through air tubes with an inner diameter of $6 \mathrm{~mm}$. The air pressure was set at $0.05 \mathrm{MPa}$ by an air regulator.

The speed of air blowing was gauged at the point $1.5 \mathrm{~mm}$ apart from the ejection outlet by a hot-wire anemometer.

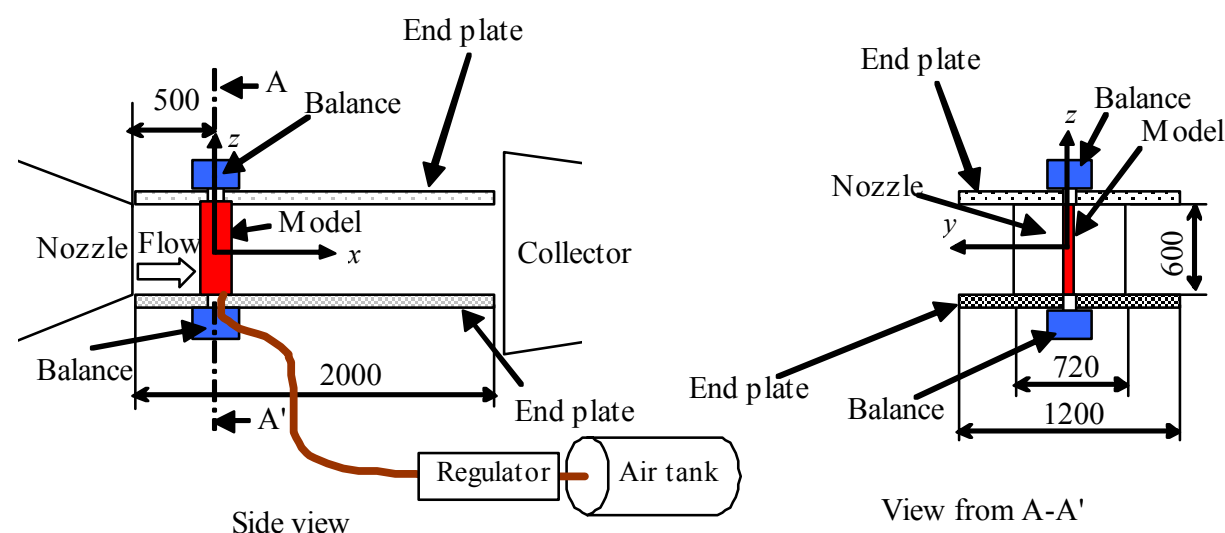

Fig. 3 Experimental apparatus for the two-dimensional panhead model (unit: mm)

\subsection{Results and discussion}

The speed of the air blowing was measured on the condition that the free stream velocity was zero and air blew only from one side of the panhead. The averaged velocity among the outlets was $5.7 \mathrm{~m} / \mathrm{s}$, which was $14 \%$ of the free stream velocity of $42 \mathrm{~m} / \mathrm{s}$.

Since the panhead shape is vertically symmetric, we only present results with the air blowing from the outlets on the bottom side of the panhead. Figure 4 exhibits the pressure distribution on the panhead model with and without the air blowing. Figure 5 explains the difference in pressure between with and without the air blowing. The transverse axis of these figures indicates the location of pressure ports by means of the angles between the free stream direction and a line that connects the pressure port with the center of the model. The locations $\theta=0^{\circ}$ and $\theta=180^{\circ}$ correspond to the leading and the trailing edges respectively. The figures indicate that the air blowing increases pressure on the bottom side of the panhead.

Table 1 proclaims the changes of the aerodynamic coefficients of the model by the air 
blowing. These values of the coefficients were obtained in two ways. One was to use the values gauged by the balances supporting the model, and the other was to integrate pressure on the model surface measured at pressure ports. The changes of the aerodynamic coefficients estimated by these two methods are almost consistent with each other. The change of the lift coefficient by the air blowing is approximately 0.04 . It is noticeable that the air blowing does not change the drag coefficient.

The conclusion drawn from the above results is that the air blowing from single side of the panhead can control its lift force. The blowing from the bottom side of the panhead increases the lift force, while the blowing from the top side decreases it.
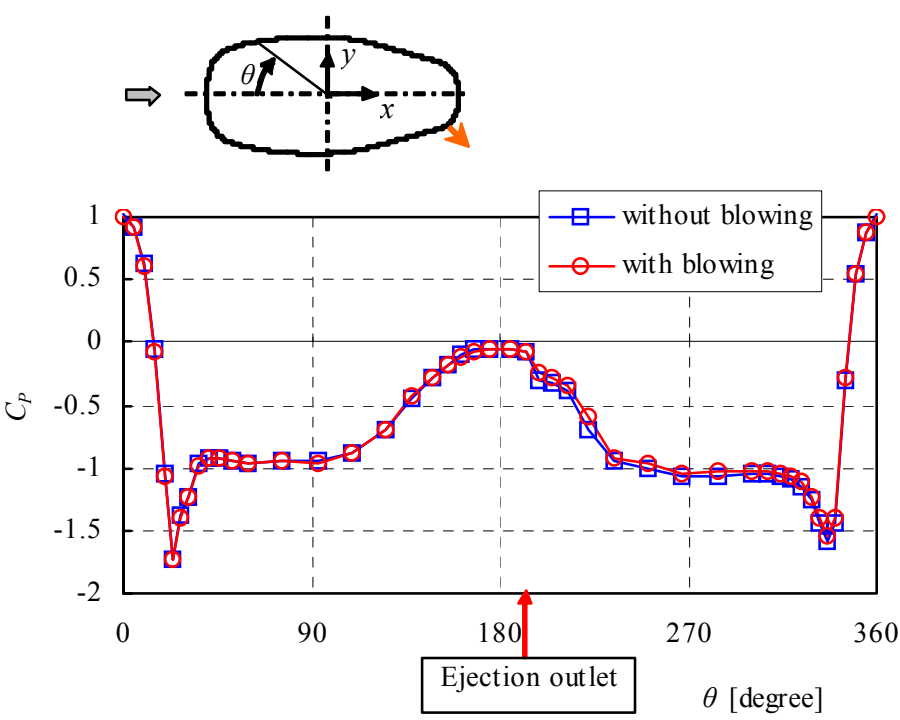

Fig. 4 Surface pressure distribution on the two-dimensional panhead

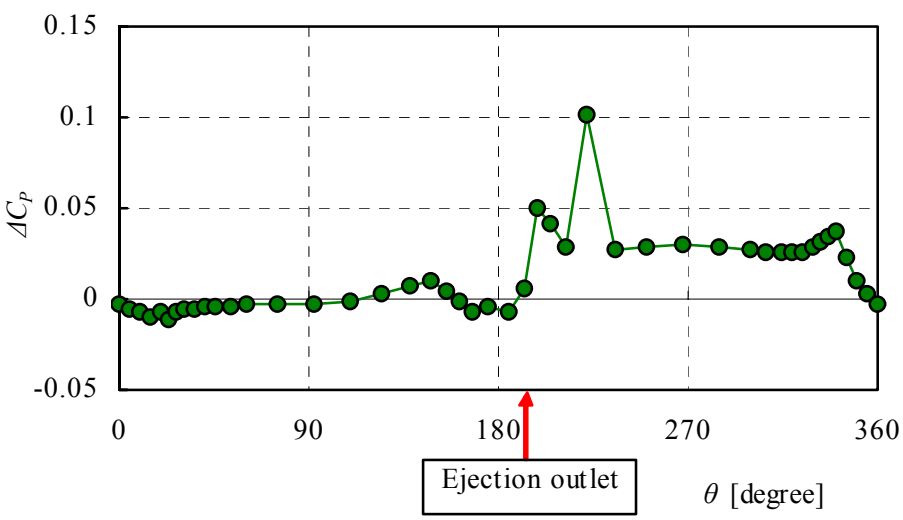

Fig. 5 Change of pressure distribution due to the air blowing

Table 1 Effect of the air blowing

\begin{tabular}{|c|c|c|}
\hline & Estimated by force data & Estimated by pressure data \\
\hline$\Delta C_{L}$ & 0.036 & 0.035 \\
\hline$\Delta C_{D}$ & -0.001 & -0.002 \\
\hline
\end{tabular}




\section{Wind tunnel test of a full-scale pantograph model}

\subsection{Full-scale pantograph model with air blowing}

To verify the ability of this technique in practical conditions, we conducted a wind tunnel test by using a full-scale pantograph model as shown in Fig. 6. A full-scale panhead model with the air blowing device was set on a single arm pantograph used for Shinkansen trains. The span length of this panhead is $900 \mathrm{~mm}$ long, and its cross section is the same shape as that of the two-dimensional one described in the previous section. The shape of the ejection outlets is also the same as that of the two-dimensional model. Since a frame installed inside the full-scale model to keep its stiffness limits its inner space, the tubes may be dented or crushed if we use the outlets at the same position of the two-dimensional model located at $\theta=167^{\circ}$ and $\theta=193^{\circ}$. Thus the positions of the outlets are changed to $\theta=148^{\circ}$ and $\theta=212^{\circ}$, respectively. In addition, the interval of the outlets in the span-wise direction is twice that of the two-dimensional model; 20 outlets are placed on each side.

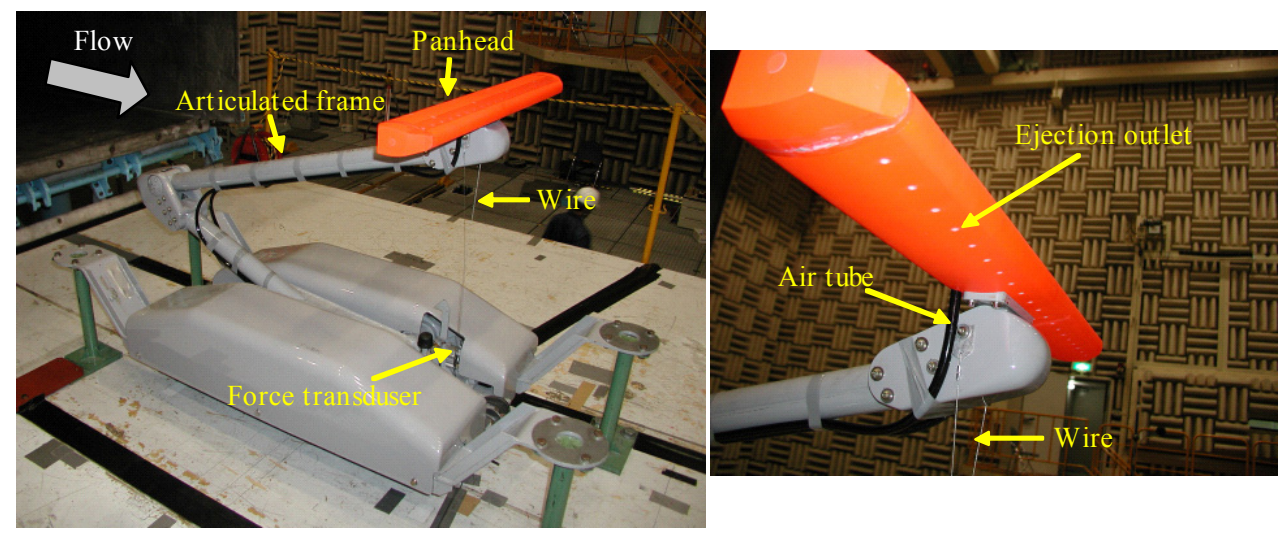

Fig. 6 Full-scale pantograph model with the air blowing

\subsection{Experimental description}

The test was carried out in a large-scale anechoic wind tunnel belonging to RTRI, which has an open-type test section of 3,000 $\mathrm{mm}$ in width and 2,500 $\mathrm{mm}$ in height. The center of the pantograph was set at the position 1,660 mm apart from the nozzle (Fig. 7).

The flow velocities were set at $42 \sim 83 \mathrm{~m} / \mathrm{s}(150 \sim 300 \mathrm{~km} / \mathrm{h})$ and the Reynolds numbers based on the chord length of the panhead were $3.2 \times 10^{5} \sim 6.4 \times 10^{5}$.

The lift force of the pantograph was evaluated by measuring the tension of a wire connecting the panhead to a base frame of the pantograph equipped with a force transducer. Compressed air (0.05 0.2 MPa) was supplied to the panhead through a pair of air tubes with a diameter of $6 \mathrm{~mm}$, which were laid along both sides of the articulated arm. These tubes were connected with an air tank of a volume of $34 l$ through an air regulator.

The velocities of the air blowing were measured at the point $1.5 \mathrm{~mm}$ apart from the ejection outlets by the hot-wire anemometer. 


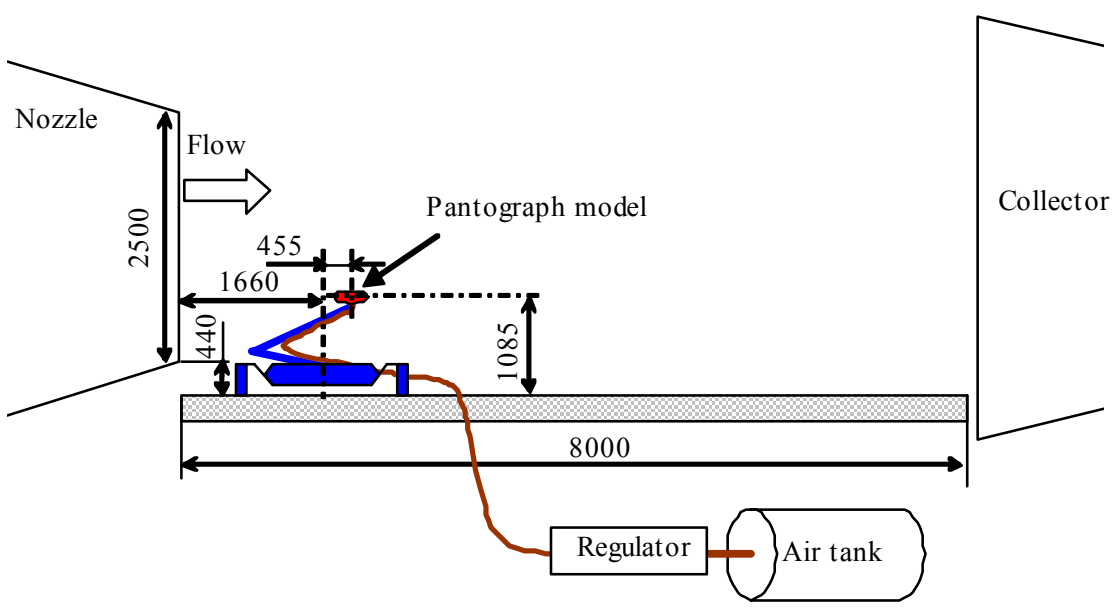

Fig. 7 Experimental apparatus for the full-scale pantograph model (unit: $\mathrm{mm}$ )

\subsection{Results and discussion}

The average of the blowing velocities at each outlet was $24.8 \mathrm{~m} / \mathrm{s}$ in the case of the fed air regulated at $0.2 \mathrm{MPa}$. While the measurement was conducted without the free stream, this value corresponds to $60 \%$ of the free stream velocity of $42 \mathrm{~m} / \mathrm{s}$.

Figure 8 indicates the lift force of the pantograph with/without the air blowing. The supplied air was regulated at $0.2 \mathrm{MPa}$. The blowing from the bottom side of the panhead increases the lift force and that from the top side decreases it. At $83 \mathrm{~m} / \mathrm{s}(300 \mathrm{~km} / \mathrm{h})$, the air blowing changes the lift force approximately by $\pm 20 \mathrm{~N}$. This value is comparable to the static uplift force of Shinkansen pantograph, which is generally set at $54 \mathrm{~N}$.

Figure 9 shows the effect of fed air pressure on the change of the lift force. The change of the lift force is almost proportional to the pressure of fed air in the region of 0.05 $\sim 0.2 \mathrm{MPa}$.

These results prove that this technique is applicable to control of the lift force of the pantograph by switching between the air blowing from the bottom side of the panhead and that from the top side, and by regulating fed air.

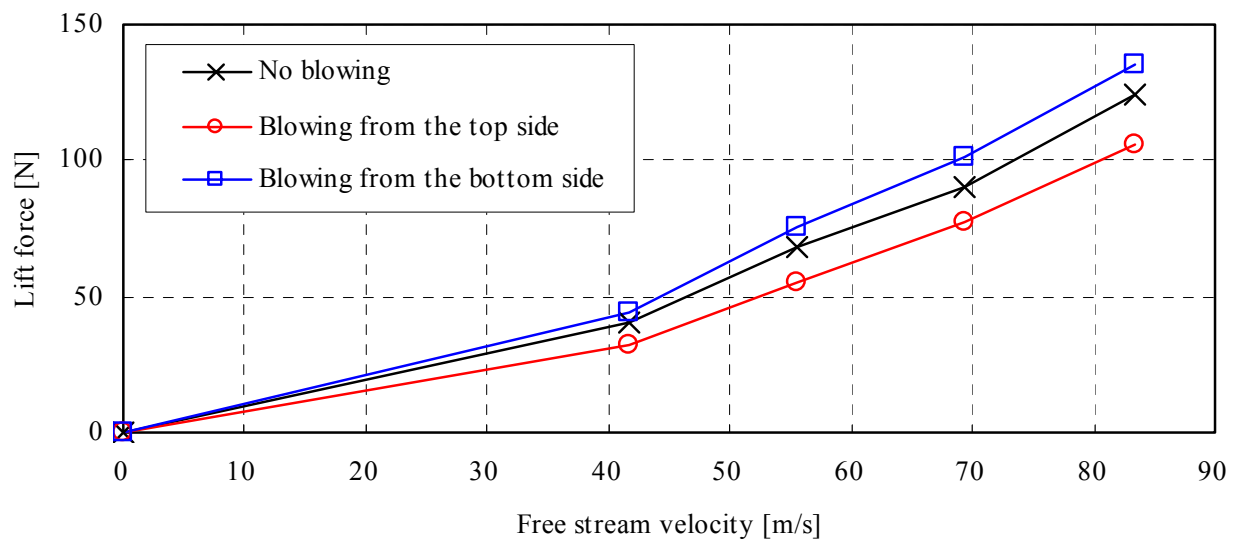

Fig. 8 Effect of the air blowing on the lift force 


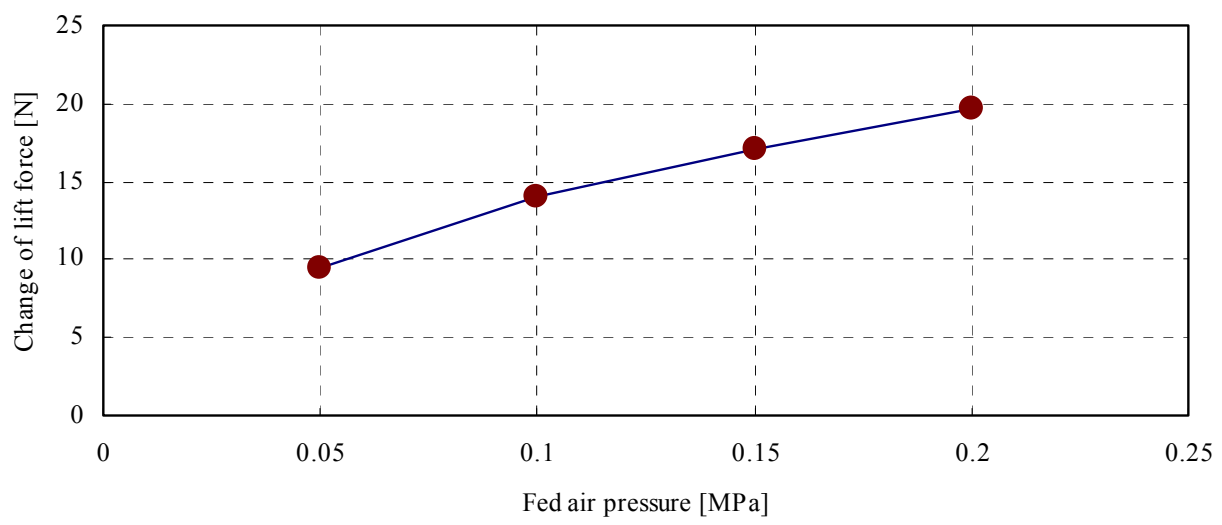

Fig. 9 Change of the lift force due to regulated fed air pressure

\section{Concluding remarks}

In this study, the air blowing was applied to the pantograph to modify its aerodynamic characteristics. First, we performed the wind tunnel test to estimate the effect of the air blowing technique by using the two-dimensional panhead model. Then we examined the full-scale pantograph model by the wind tunnel test. The experiments prove that the proposed method can control the lift force acting on the pantograph successfully.

This study was supported by Messrs. Houzu, Ito and Bukhari, the former students of Tokyo University of Agriculture and Technology. We would like to express our appreciation to them.

\section{References}

(1) Ikeda, M., Measures against pantograph noise caused by the Shinkansen, Rail International, (1997-5), pp. 35-43.

(2) Lumley, J. and Blossey, P., Control of turbulence, Annual Review of Fluid Mechanics, Vol. 30, (1998), pp. 311-327.

(3) Kim, J., Hahn, S., Kim, J., Lee, D., Choi, J., Jeon, W. and Choi, H., Active controls of turbulent flow over a model vehicle for drag reduction, Journal of Turbulence, Vol. 5, (2004).

(4) Wygnanski, I., On active control of separation from bluff bodies, on CD-ROM of Proceedings of International Conference on Jets, Wakes and Separated Flows, (2005).

(5) Suzuki, M., Ikeda, M. and Yoshida, K, Study on numerical optimization of cross-sectional panhead shape for high-speed train, Journal of Mechanical Systems for Transportation and Logistics, Vol. 1, No. 1, (2008), pp. 100-110. 